\title{
Deformation of nanotubes in peeling contact with flat substrate: An in situ electron microscopy nanomechanical study
}

\author{
Xiaoming Chen, ${ }^{1}$ Meng Zheng, ${ }^{1}$ Qing Wei, ${ }^{1}$ Stefano Signetti, ${ }^{2}$ Nicola M. Pugno, ${ }^{2,3,4}$ \\ and Changhong $\mathrm{Ke}^{1, \mathrm{a})}$ \\ ${ }^{1}$ Department of Mechanical Engineering, State University of New York at Binghamton, Binghamton, \\ New York 13902-6000, USA \\ ${ }^{2}$ Laboratory of Bio-Inspired and Graphene Nanomechanics, Department of Civil, \\ Environmental and Mechanical Engineering, University of Trento, Trento, Italy \\ ${ }^{3}$ Centre for Materials and Microsystems, Fondazione Bruno Kessler, Povo (Trento), Italy \\ ${ }^{4}$ School of Engineering and Materials Science, Queen Mary University of London, London, United Kingdom
}

(Received 8 January 2016; accepted 31 March 2016; published online 19 April 2016)

Peeling of one-dimensional (1D) nanostructures from flat substrates is an essential technique in studying their adhesion properties. The mechanical deformation of the nanostructure in the peeling experiment is critical to the understanding of the peeling process and the interpretation of the peeling measurements, but it is challenging to measure directly and quantitatively at the nanoscale. Here, we investigate the peeling deformation of a bundled carbon nanotube (CNT) fiber by using an in situ scanning electron microscopy nanomechanical peeling technique. A precalibrated atomic force microscopy cantilever is utilized as the peeling force sensor, and its back surface acts as the peeling contact substrate. The nanomechanical peeling scheme enables a quantitative characterization of the deformational behaviors of the CNT fiber in both positive and negative peeling configurations with sub-10 nm spatial and sub- $\mathrm{nN}$ force resolutions. Nonlinear continuum mechanics models and finite element simulations are employed to interpret the peeling measurements. The measurements and analysis reveal that the structural imperfections in the CNT fiber may have a substantial influence on its peeling deformations and the corresponding peeling forces. The research findings reported in this work are useful to the study of mechanical and adhesion properties of 1D nanostructures by using nanomechanical peeling techniques. Published by AIP Publishing. [http://dx.doi.org/10.1063/1.4945995]

\section{INTRODUCTION}

One-dimensional (1D) nanostructures, such as nanotubes and nanowires, possess many extraordinary structural and physical properties and are ideal building blocks for various materials and systems such as composites, sensors, and electronics. Due to their large surface-to-volume ratio characteristics, the interface plays an important role in their structural and functional properties, which impacts many of their applications. For example, the adhesion between carbon nanotubes (CNTs) and substrates may induce substantial transverse deformations in CNTs, thus alternating their band-gaps and impacting many of their electronics applications. ${ }^{1,2}$ Therefore, the interfacial binding or adhesion properties of nanotubes with substrates have been intensively studied by using a variety of theoretical/computational and experimental techniques. ${ }^{3-12}$ Direct and quantitative experimental measurements of nanotube-substrate adhesion interactions demand the mechanical separation or peeling of individual nanotubes from the contacting substrates. The simultaneous measurements of the nanotube peeling deformation and the corresponding peeling force with adequate spatial and force resolutions are quite challenging at the nanoscale. Therefore, the reported experimental studies in

\footnotetext{
a) Author to whom correspondence should be addressed. Electronic mail: cke@binghamton.edu.
}

the literature remain quite limited. Some of the notable works are briefly summarized below.

Strus et al. investigated the nanotube adhesion by performing nanomechanical peeling measurements inside an atomic force microscope (AFM). ${ }^{4,6}$ In their measurements, a CNT was attached to a tipless AFM cantilever. The CNT was controlled to first make contact with the substrate and then was gradually lifted from the contacting substrate. The vertical peeling force and the displacement of the nanotube fixed end that was attached to the AFM cantilever were measured through recording the deflection of the AFM cantilever using a laser reflection scheme. The study revealed multiple stable states in the peeling deformation of the CNT in the forms of line or point contact with the substrate. It is noted that the exact mechanical deformation curvature of the tested CNT during the peeling test was not directly measured due to the lack of visualization means in the employed peeling technique. Therefore, the interpretation of the experimental measurements relied on the continuum modeling of the CNT deformation based on idealized CNT structures. Ishikawa and his colleagues reported an in situ nanomechanical peeling study of CNT adhesion with a graphite substrate inside a scanning electron microscope (SEM). ${ }^{5,13}$ A CNT, which was attached to a self-sensing AFM cantilever, was placed horizontally and controlled to first approach and make contact with, and then peel away from, a vertically placed flat graphite substrate. The peeling deformation of the 
CNT was measured directly using the electron beam, while the corresponding peeling force was recorded from the reading of the self-sensing AFM cantilever. Roenbeck et al. ${ }^{7}$ reported an in situ SEM nanomechanical peeling study on the adhesion between CNTs and graphene substrates by using a pre-calibrated AFM cantilever.

It is noted that the mechanical deformation of CNTs in the peeling test plays an important role in the understanding of the peeling process and in the interpretation of the peeling measurement. The aforementioned peeling measurements were interpreted by means of theoretical modeling and/or computational simulations based on idealized CNT structures. The direct visualization of the mechanical deformation curvature of CNTs enables a quantitative characterization of their mechanical response to the applied peeling force, in particular, those delicate details caused by structural imperfections. Prior studies have revealed that CNTs may possess a variety of structural defects, e.g., Stone-Wales transformation, ${ }^{14}$ which comes into being during either synthesis or post-processing stages and substantially affects their structural and mechanical properties. ${ }^{15-17}$ Therefore, it is of importance to understand the role of structural nonidealities in CNTs in the peeling experiments, which, however, remains unexplored. This is in part due to the technical challenges associated with the high-resolution visualization of nanoscale deformation curvature of the CNT in the peeling measurement, which has so far only been reported by few research groups in the world.

In this paper, we investigate the mechanical deformation of CNTs in peeling contact with flat substrates using an in situ SEM nanomechanical peeling scheme, as illustrated in Figure 1(a). In this peeling scheme, the tested nanotube is mounted to a nanomanipulator probe and placed horizontally in front of, or in peeling contact with, the back surface of a vertically placed AFM cantilever. Both the peeling

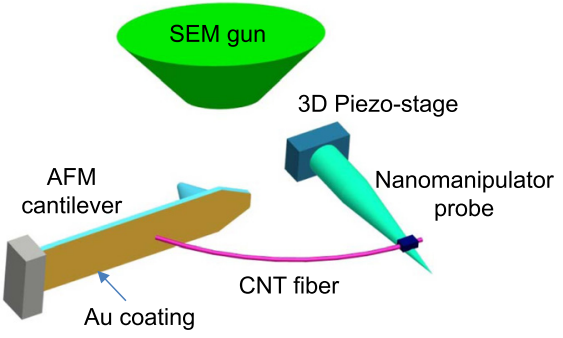

(a)

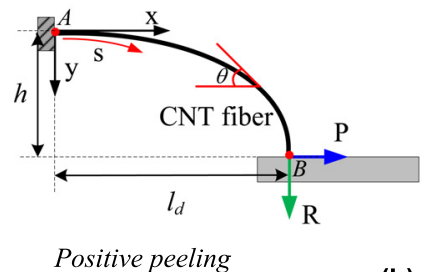

Positive peeling

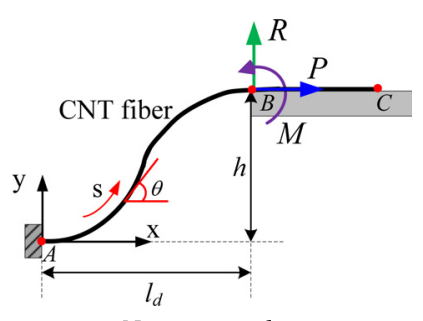

(b)

Negative peeling
FIG. 1. (a) 3D schematic of in situ electron microscopy nanomechanical peeling of a CNT fiber from the Au-coated back surface of a vertically placed AFM cantilever inside a high resolution scanning electron microscope. (b) Schematics of positive and negative peeling configurations and the corresponding nonlinear mechanics models. deformation of the nanotube and the deflection of the AFM cantilever caused by the peeling force stay in the horizontal plane and perpendicular to the electron beam and thus can be measured precisely using the electron beam with a spatial resolution of a few nanometers. A unique aspect of this peeling scheme compared with those employed in prior studies ${ }^{5,7,13}$ is the integration of the force sensor with the contacting substrate. The AFM cantilever acts as both the force sensor in sensing the peeling force along the direction perpendicular to the contacting surface while its back surface acts as the contacting substrate with the nanotube. It is noted that the cantilever back surface can be coated with a variety of materials, such as metals, polymers, and ceramics. Therefore, this peeling scheme can be used to study the binding/adhesion interaction between nanotubes (or nanowires) and different types of material surfaces. Another unique aspect of our nanomechanical peeling technique is that it allows quantitative studies of the peeling deformation of CNTs under different stable equilibrium states. For the previously reported peeling studies, ${ }^{4-7,13}$ the CNT is always peeled from the flat substrate by a force that forms a zero or acute angle with the normal direction of the substrate. The deformed CNT stays entirely above the contacting substrate surface as illustrated by the left drawing in Figure 1(b). This peeling configuration is denoted here as peeling at a positive angle or positive peeling. Under a peeling force that forms an obtuse angle with the normal direction of the substrate, the CNT either entirely conforms to the substrate surface or is pulled downward along the substrate edge, as displayed by the right drawing in Figure 1(b). The latter peeling configuration is denoted as peeling at a negative angle or negative peeling. To date and to the best of our knowledge, the stable equilibrium state of nanotubes in negative peeling configuration has not been explored in experiment. Our nanomechanical peeling scheme as illustrated in Figure 1(a) uniquely enables a study of the mechanical deformations of individual CNTs in both peeling configurations.

\section{RESULTS AND DISCUSSION}

\section{A. In situ SEM nanomechanical peeling experiments}

The nanomechanical peeling measurements were performed in situ inside a high resolution SEM (FEI Nanolab 600). Silicon AFM cantilevers (CSG 01, NT-MDT) with a 5-nm-gold $\mathrm{Au}$ ) coating on their back surfaces were employed. The AFM cantilevers have a nominal spring constant of $0.03 \mathrm{~N} / \mathrm{m}$ and a cantilever width of $30 \mu \mathrm{m}$. The spring constant of each employed AFM cantilever was calibrated using thermal tuning methods. ${ }^{18}$ One representative nanomechanical peeling experiment is presented in Figure 2. Figure 2(a) shows that a CNT fiber was attached to a nanomanipulator probe and was controlled to be placed parallel to the back surface of a pre-calibrated AFM cantilever (spring constant of $0.064 \mathrm{~N} / \mathrm{m}$ ). The AFM cantilever was vertically mounted to the SEM stage. The manipulator probe was attached to a 3D closed-loop piezo-stage with $1 \mathrm{~nm}$ motion resolution in all axes. ${ }^{19}$ The CNT fiber was obtained through nanomanipulation of thin-bundled single-walled CNTs that were transferred to copper transmission electron 

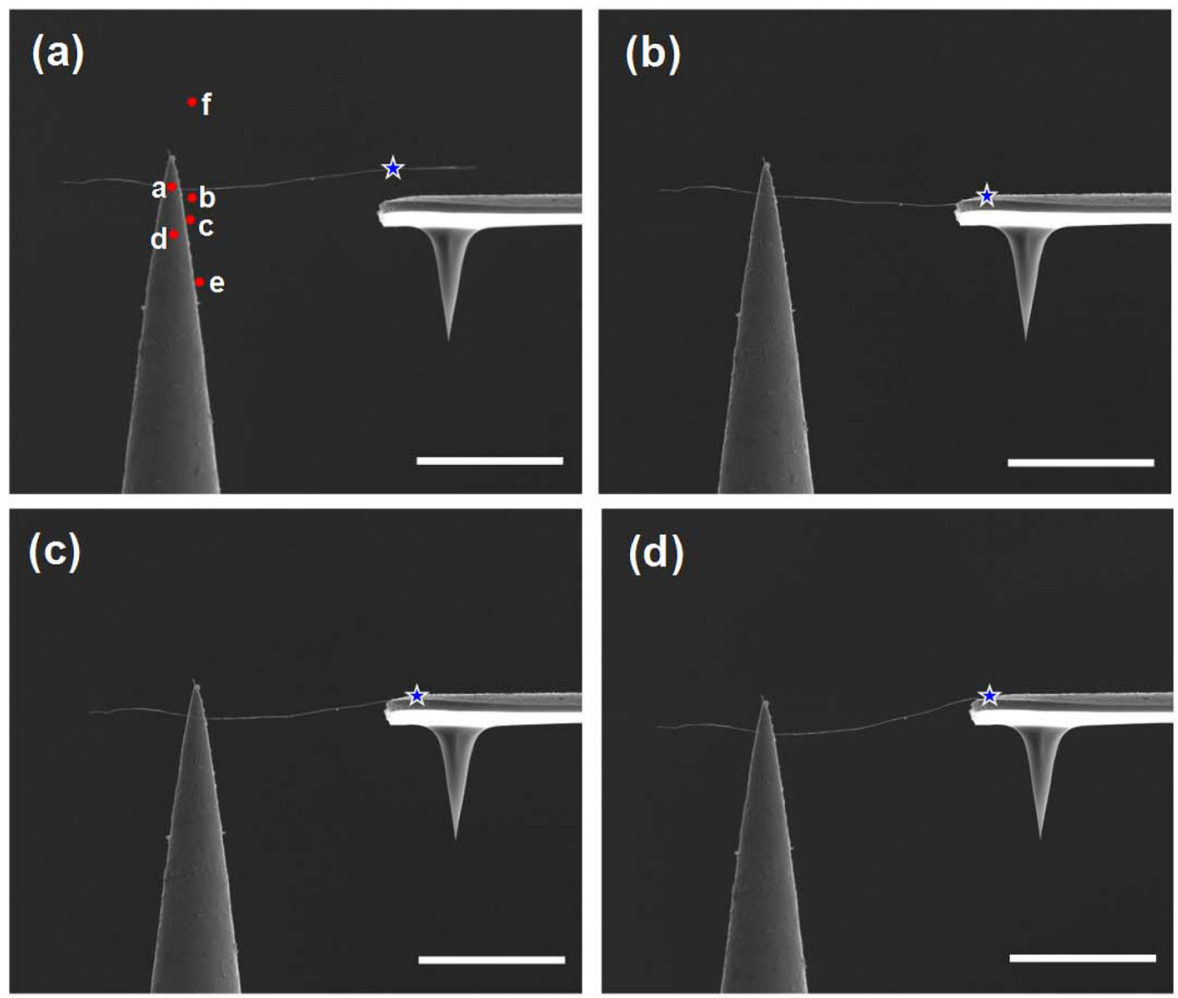

FIG. 2. Selected SEM snapshots of the original CNT fiber (a) and its mechanical deformations at various peeling positions (b)-(f). The dashed box in (f) is a zoom-in view of the defect site that is indicated by the white arrow. The red dots in (a) mark the positions of the attached point of the CNT fiber in the peeling states displayed in (a)-(f). The stars in (a)-(f) mark the defect site on the CNT fiber. All scale bars represent $10 \mu \mathrm{m}$.
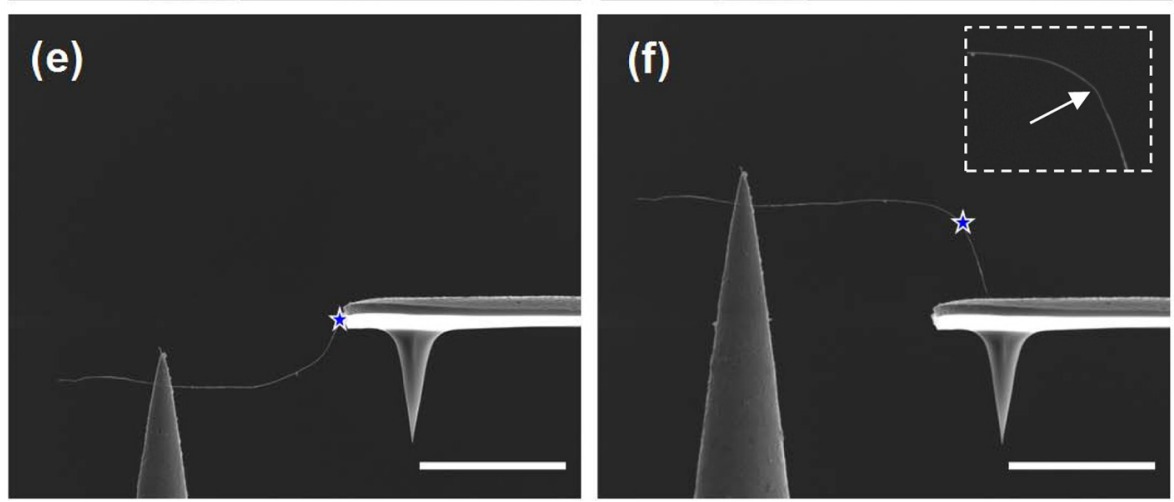

microscope (TEM) grids from a silicon wafer where they were vertically grown using chemical vapor deposition (CVD) methods. ${ }^{20}$ The bundled CNT fibers obtained using this protocol were previously characterized using high resolution transmission electron microscopy (HRTEM) ${ }^{20,21}$ and were shown to be formed by parallel and tightly bound CNTs. Electron beam induced deposition (EBID) of carbon was used to enhance the attachment of the CNT fiber to the nanomanipulation probe. $^{22}$ The length of the CNT fiber, which is defined as the distance between its attached point to the probe to its right-side free end, and its transverse width, were measured to be about $20.5 \mu \mathrm{m}$ and $45 \mathrm{~nm}$, respectively.

Figures 2(b)-2(f) show five selected high resolution SEM snapshots, in which the deformation curvatures of the CNT fiber in different peeling contact positions with the substrate are exhibited. To assist the visualization of the motion of the manipulator probe during the peeling experiments, the respective positions of the fixed point of the CNT fiber in the peeling tests displayed in Figures 2(a)-2(f) are marked by red dots in Figure 2(a). The manipulator probe was controlled to move in a downward direction on the SEM images from Figures 2(a) to 2(e), while in an upward move from
Figures 2(e) to 2(f). Figure 2(b) shows that the CNT fiber was pulled down to stay at approximately the same horizontal level (on the image) with the substrate surface, with a nanotube segment of $7.9 \mu \mathrm{m}$ in length staying on and conforming to the substrate surface.

By controlling the motion of the manipulator probe, the CNT fiber was peeled at a negative angle and deformed into an $S$ shape, as displayed by the deformation curvatures shown in Figures 2(c)-2(e). It can be seen that the curvature change of the deformed CNT fiber was accompanied with a gradual pull-off of the tube segment that stayed on the substrate surface. The length of the tube segment that stayed on the substrate surface decreased to $5.8 \mu \mathrm{m}$, as measured from Figure 2(e). By moving the fixed point of the CNT fiber upward, a substantial conformation transition occurred in the peeling deformation of the CNT fiber. Figure 2(f) shows the deformation curvature of the CNT fiber under positive peeling with its fixed point placed $6.4 \mu \mathrm{m}$ above the substrate. The exhibited arc shape of the deformed CNT fiber indicates a point contact between the free end of the CNT fiber and the substrate. The seeming discontinuity in the slope of its deformation curve at the position of about $6.0 \mu \mathrm{m}$ from its free 
end, as indicated by the white arrow in the zoom-in view of Figure 2(f), implies the presence of prominent structural defects. For the purpose of visualization, the defect site is marked with a star on the CNT fiber displayed in Figures 2(a)-2(f). The seeming structural defects in the tested CNT fiber can be attributed to one or multiple possible sources: (1) defects formed during the synthesis stage; (2) defects formed and/or enhanced during the post-processing stage, including the transfers of the bundled tubes from the growing substrate to the TEM copper grid and then to the manipulator probe; (3) defects caused and/or enhanced by the electron beam irradiation. The defects formed during the tube synthesis are likely distributed on a tube in a random manner. The transferring and manipulation/peeling processes can result in defects at particular sites, which may be further enhanced through a long-time exposure (about $10 \mathrm{~h}$ ) to the electron beam. Because the whole CNT fiber was exposed to the electron beam during the experiment, the irradiation-induced tube damages were likely distributed along the whole fiber. Our analysis of the recorded SEM images shows no visible changes to the topography of the tested CNT fiber, such as its length and width, from the moment after it was transferred to the TEM copper grid to the end of the peeling experiments. Therefore, it is highly unlikely that the observed structural defects were caused by the electron beam irradiation along.

It is noted that the SEM images only display the CNT fiber displacement in the horizontal direction that is perpendicular to the electron beam. Our analysis shows that the CNT fiber length remains largely unchanged (less than $1 \%$ in deviation) at those deformation positions displayed in Figure 2, indicating that the CNT fiber was placed in a nearly horizontal position. This is also consistent with the fact that the whole CNT fiber was in good focus at the same focusing length under the SEM electron beam. Therefore, the measured CNT fiber deflection from the SEM images can be considered as a reasonable approximation to its actual deformation.

In the peeling experiments, the vertical component of the contact force between the free end of the CNT fiber and the substrate induced a deflection of the AFM cantilever. Therefore, the contact force can be measured through quantifying the deflection of the AFM cantilever based on the recorded SEM images. It is noted that quite a small deflection of the AFM cantilever was observed in the peeling experiments. Here, we quantify the AFM cantilever deflection through digitally processing the recorded SEM images with the aid of digital imaging correlation (DIC) techniques. $^{23}$ The pixel resolution of the recorded SEM images is measured to be $25 \mathrm{~nm} /$ pixel. By using DIC, a displacement measurement resolution of $2.5 \mathrm{~nm}$, which corresponds to one-tenth of a pixel, can be achieved. The force measurement resolution and uncertainty of the nanomechanical peeling technique by using the AFM cantilever displayed in Figure 2 are calculated to be $0.16 \mathrm{nN}$ and $0.08 \mathrm{nN}$, respectively. The vertical peeling force, which is denoted as $R$ in Figure 1(b), is measured for the peeling positions displayed in Figures 2(c)-2(f), and the values are listed in Table I. The stable equilibrium peeling position with the CNT fiber that stays in an S-shape and in a line contact with the substrate as shown in Figure 2(e) is stabilized by a vertical peeling force of $0.64 \mathrm{nN}$, which defects the AFM cantilever in a downward direction. For the peeling position with the CNT fiber staying in an arc-shape and in a point contact with the substrate as shown in Figure 2(f), the corresponding vertical peeling force is a pulling force in nature and is found to be smaller than the force measurement resolution limit $(0.16 \mathrm{nN})$. The results indicate that the peeling deformation of the CNT fiber and the corresponding peeling force are substantially dependent on the peeling configuration.

\section{B. Nonlinear continuum mechanics (NCM) model of the CNT fiber peeling deformations}

The nanomechanical peeling experiments of the CNT fiber in various peeling contacts with the substrate surface are interpreted using an NCM model. The SEM images clearly show that the deformation of the CNT fiber is in the large displacement regime. Therefore, the CNT fiber is simplified as a uniform non-extensible elastica $\operatorname{rod}^{24}$ with a circular cross-section. This modeling simplification is consistent with the prior experimental findings that nanotubes could be repeatedly deformed to large bending angles and strain without permanent alteration of the tube topography. ${ }^{25}$ Our recent experimental work also demonstrated the elastica-like behavior for bundled CNTs in the large displacement regime. ${ }^{20,21}$ The mechanical deformations of the rod in two peeling configurations are illustrated in Figure 1(b), and its deformation curvature is governed by

$$
E I \frac{\mathrm{d}^{2} \theta}{\mathrm{d} s^{2}}+P \sin (\theta)-R \cos (\theta)=0,
$$

where $E$ and $I=\pi d^{4} / 64$ are the Young's modulus and moment of inertia of the rod ( $d$ is the diameter of the rod), respectively; $s$ is the arc length along the deformed rod; $\theta$ is the angle between the tangent of the rod at $s$ and $x$-axis; and $R, P$, and $M$ represent the vertical and horizontal forces and the bending moment that act on the rod cross-section at the contact between the end of the deformed rod segment and the substrate, i.e., point $B$ in Figure 1(b), respectively. The boundary conditions for positive peeling configuration are considered as a fixed end at point $A$ and a pin support at point $B$, and are given as $x_{A}=y_{A}=0, \theta_{A}=0, x_{B}=l_{d}, y_{B}=h$, $\left.\frac{\mathrm{d} \theta}{\mathrm{d} s}\right|_{B}=0$. Here, $h$ is the deflection of the rod at its free end, and $l_{d}$ is the horizontal spanning width of the deformed rod segment. Both $h$ and $l_{d}$ can be measured directly from the recorded SEM images. For negative peeling configuration, the boundary conditions include two fixed ends at points $A$ and $B$ and a flat segment $B C$ and are given as $x_{A}=y_{A}=0$, $x_{B}=l_{d}, y_{B}=y_{C}=h$, and $\theta_{A}=\theta_{B}=\theta_{c}=0$. By integration of Eq. (1), the curvature of the deformed rod is given as

$$
\frac{\mathrm{d} \theta}{\mathrm{d} s}= \pm \sqrt{2\left(\frac{\sqrt{P^{2}+R^{2}}}{E I}\right)^{2}\left(\cos (\theta-\alpha)-\cos \left(\theta_{B}-\alpha\right)\right)+\left(\left.\frac{\mathrm{d} \theta}{\mathrm{d} s}\right|_{B}\right)^{2}}
$$

where $\alpha=\tan ^{-1}(R / P)$. It is noted that the length of the deformed rod segment in the point peeling contact, as 
TABLE I. List of the experimentally (exp) measured CNT fiber peeling deformations and forces, and their comparisons with the theoretical predictions based on NCM models and FEM simulations.

\begin{tabular}{|c|c|c|c|c|c|c|c|c|}
\hline \multirow{3}{*}{$\begin{array}{l}\text { Peeling } \\
\text { position in Figure } 2\end{array}$} & \multicolumn{4}{|c|}{ CNT fiber deformation $(\mu \mathrm{m})$} & \multicolumn{4}{|c|}{ Contact/peeling force $(\mathrm{nN})$} \\
\hline & \multirow{2}{*}{$\begin{array}{l}\text { Maximum } \\
\text { deflection } h(\exp )\end{array}$} & \multirow{2}{*}{$\begin{array}{l}\text { Horizontal spanning } \\
\text { width } l_{d}(\exp )\end{array}$} & \multicolumn{2}{|c|}{ Deformed length $L$} & \multirow[b]{2}{*}{$R(\exp )$} & \multirow[b]{2}{*}{$R(\mathrm{NCM})$} & $R$ & \multirow[t]{2}{*}{$P$} \\
\hline & & & $\exp$ & $\mathrm{NCM}$ & & & (FEM) & \\
\hline $\mathrm{c}$ & 1.21 & 12.62 & 12.88 & 12.75 & $0.32 \pm 0.08$ & 0.28 & 0.35 & 0.05 \\
\hline $\mathrm{d}$ & 2.43 & 14.15 & 14.51 & 14.40 & $0.45 \pm 0.08$ & 0.41 & 0.51 & 0.17 \\
\hline e & 4.94 & 11.98 & 14.68 & 13.16 & $0.64 \pm 0.08$ & 1.21 & 0.73 & 0.68 \\
\hline $\mathrm{f}$ & 6.39 & 16.25 & 20.5 & 20.5 & $<0.16$ & 0.10 & -0.03 & -0.07 \\
\hline
\end{tabular}

displayed in Figure 2(f), equals the original rod length. For the line peeling contact, as displayed in Figures 2(c)-2(e), the length of the deformed rod segment is less than the original rod length and is given as $L=\int_{A}^{B} \mathrm{~d} s$.

By using the above NCM model, we analyze the mechanical deformation of the CNT fiber in the peeling positions shown in Figures 2(c)-2(f) and compare the theoretical predictions with experimental measurements. The results are presented in Figure 3 and Table I. In our theoretical analysis, $E=197.5 \mathrm{GPa}$ is employed based on the reported tensile

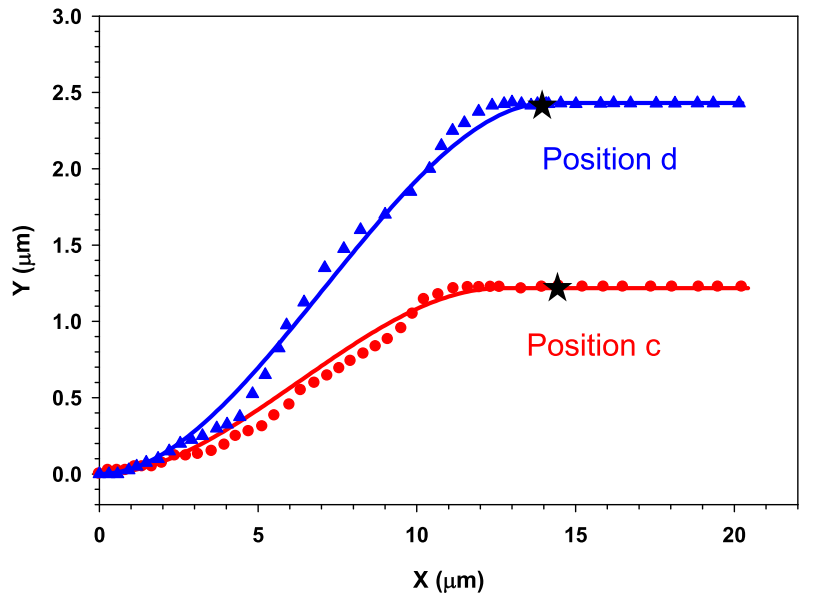

(a)

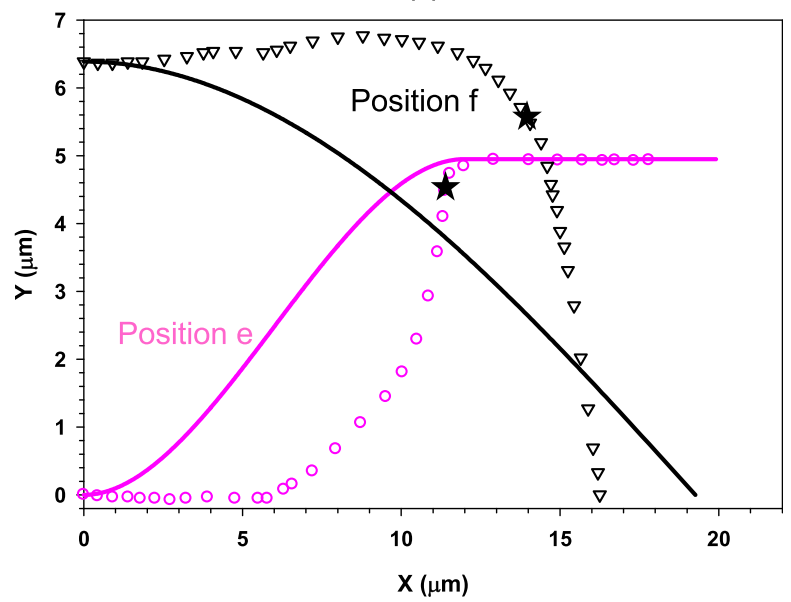

(b)

FIG. 3. Comparison of the experimental measurements (dotted lines) and the NCM-based theoretical predictions (solid lines) on the deformation curvatures of the CNT fiber: (a) for the two peeling positions shown in Figures 2(c) and 2(d); (b) for the two peeling positions shown in Figures 2(e) and 2(f). The stars mark the position of the defect site on the CNT fiber. testing data of similar CNT fibers. ${ }^{26}$ By assuming a frictionfree contact on the CNT-substrate interface, the horizontal reaction force $P$ is set as zero for both peeling configurations. Figure 3(a) shows the comparison of the theoretical predictions and experimental measurements of the CNT deformation for the two peeling positions shown in Figures 2(c) and 2(d), which displays a reasonably good agreement. It is noted that the defect site on the CNT fiber, which is marked as a star, stays either on the flat segment that conforms to the flat substrate (red curve) or close to the fixed-end (blue curve) and thus has a limited impact on the CNT deformation. However, substantial discrepancies occur in the comparison of the theoretical predictions and experimental measurements of the CNT deformation for the two peeling positions shown in Figures 2(e) and 2(f), which is exhibited in Figure 3(b). The results clearly show that the structural defects in CNTs may have a significant influence on its peeling deformations, in particular, when the defect sites are located in the central position of the deformed nanotube segment. Similar phenomena are also observed in the comparison of the theoretically predicted and experimentally measured peeling forces, which are listed in Table I. The corresponding peeling forces for the positions shown in Figures 2(c) and 2(d) are predicted to be 0.28 and $0.41 \mathrm{nN}$, respectively, both of which are close to the experimental values $(0.32$ and $0.45 \mathrm{nN})$. For the peeling position shown in Figure 2(e), the theoretically predicted peeling force is $1.21 \mathrm{nN}$ and is substantially higher than the experimental value $0.64 \mathrm{nN}$. The theoretically predicted peeling force for the peeling position shown in Figure 2(f) is merely $0.10 \mathrm{nN}$, which is below the force measurement resolution limit of the DICaided imaging processing technique. The data explain the peeling force measurement results wherein the peeling force in the point-contact peeling configuration is too small to be detected. Table I also includes the comparison of the experimentally measured and theoretical predicted values of the deformed CNT fiber length $L$. It can be seen that substantial discrepancies occur only for the peeling position shown in Figure 2(e), which can be also attributed to the effect of the structural defects in the CNT fiber. All these results clearly show that the structural defects in CNTs may have a substantial influence not only on their peeling deformation but also on the peeling force.

\section{Numerical simulations of the CNT fiber peeling deformations}

In addition to the theoretical analysis based on the NCM model, we perform finite element method (FEM)-based 
numerical simulations to better understand the mechanical deformations of the CNT fiber in the peeling measurements, in particular, the role of the structural defect. In order to compare the FEM numerical results with the actual experimental measurements of the CNT fiber deformation at different peeling positions, the undeformed CNT fiber geometry is first traced from the acquired SEM image (Figure 2(a)) via a CAD software. The overall CNT fiber length of $28.1 \mu \mathrm{m}$ (including the undeformed CNT portion on the left-side of the manipulator probe) is discretized with elements of $75 \mathrm{~nm}$ in length, which thus results in 374 elements in total. This length has been verified to be consistent for all the acquired SEM images. The FEM simulations are performed based on the same CNT fiber diameter and Young's modulus that are used in the NCM modeling. The structural defects at the marked defect site in the CNT fiber are simplified and modeled here as a short CNT segment (two elements with a total length of $150 \mathrm{~nm}$ ) with a reduced cross-section. It is noted that the structural defects in the tested CNT fiber may exist in a much more sophisticated form and are likely intractable to elucidate precisely using simple models. Nonetheless, the adoption of the simplified model here regarding the structural defects in the CNT fiber will help us to better understand how the structural nonidealities affect its peeling behavior. In order to estimate the magnitude of the defect, the diameter of these two elements is progressively reduced for a minimization of the difference between the experimentally measured deformation profile and the simulation derived geometry in the positive peeling configuration as displayed in Figure 2(f). A defect segment of diameter $d^{*}=18 \mathrm{~nm}$ is identified to produce best-fit deformation curves to the measurements. Figure 4 shows the superposition of the predicted deformation profiles based on the FEM simulations and the SEM snapshots for three selected peeling positions, which displays a good agreement for all. The FEM simulations also provide the contact/peeling forces between the CNT fiber and the substrate for the peeling positions shown in Figures 2(c)-2(f), which are listed in Table I. The vertical component of the peeling force $R$ is found to be $-0.03 \mathrm{nN}$ via the FEM simulations for the peeling position shown in Figure 2(f) and is beyond the force measurement

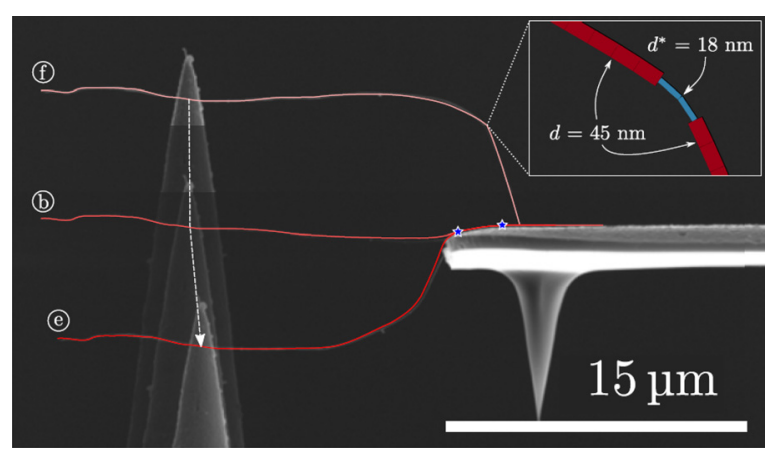

FIG. 4. Superposition of experimental measurements and finite element simulations for three selected CNT fiber deformation profiles displayed in Figures 2(b), 2(e), and 2(f). The finite element simulations are based a CNT fiber with a defect segment of $18 \mathrm{~nm}$ in diameter and $150 \mathrm{~nm}$ in length. The inset image shows the mesh detail at the defect site of the deformed fiber under positive peeling. resolution limit of the employed nanomechanical peeling scheme, which is consistent with both the experimental and the NCM modeling results. For the peeling position shown in Figure 2(e), the FEM simulations reveal a value of 0.73 $\mathrm{nN}$ for $R$, which is close to the measurement value $(0.64$ $\mathrm{nN}$ ), but is substantially lower than the NCM-predicated value $(1.21 \mathrm{nN})$. The discrepancy can be attributed to the fact that the structural defect in the CNT fiber is taken into account in the FEM simulations, while not in the NCM prediction. The FEM simulations results further confirm the importance of the structural defects in CNTs in the study of peeling deformation and in the adhesion study using peeling techniques. Without properly accounting for the effect of the structural defects in CNTs, substantial errors may occur in the theoretical prediction of CNT peeling deformations and/ or in the interpretation of the peeling measurements based on idealized CNT structures. We want to emphasize here that the structural defects, such as the ones in the tested CNT fiber, may not be visible by means of pre-test inspection using the high resolution electron beam. Therefore, high resolution deformation measurements during the peeling test are essential to capture the presence and positions of the defects and to the accurate interpretation of the peeling measurement results, and it should be an integral component of the nanoscale peeling techniques.

\section{CONCLUSION}

In this paper, we investigate the peeling deformation of a CNT fiber with a flat substrate surface in both positive and negative peeling configurations by using in situ SEM nanomechanical peeling techniques in conjunction with NCM modeling and FEM simulations. The unique integration of the force sensor and the contacting substrate via a precalibrated AFM cantilever enables the high resolution measurements of the peeling deformation of the CNT fiber and the peeling forces at different peeling configurations and positions. The experimental measurements and theoretical predictions/computational simulations show that the peeling deformation of the CNT fiber and the corresponding peeling force have a strong dependence on the peeling configuration. The results also clearly reveal that structural defects in CNTs may have a substantial influence on both their peeling deformations and the corresponding peeling force. The research findings reported in this work are useful to the future study of mechanical and adhesion properties of 1D nanostructures by using nanomechanical peeling techniques.

\section{ACKNOWLEDGMENTS}

This work was supported by the U.S. Air Force Office of Scientific Research Low Density Materials Program under Grant Nos. FA9550-11-1-0042 and FA9550-15-1-0491 and the American Chemical Society Petroleum Research Fund and was partially supported by the U.S. National Science Foundation under Grant No. CMMI-1537333. The in-situ SEM nanomechanical measurements were performed using the facilities in the Analytical and Diagnostics Laboratory at Binghamton University's Small Scale Systems Integration and Packaging Center $\left(S^{3} I P\right)$. N.M.P. was supported by the 
European Research Council (ERC StG Ideas 2011 BIHSNAM No. 279985, ERC PoC 2013 KNOTOUGH No. 632277, ERC PoC 2015 SILKENE No. 693670) and by the European Commission under the Graphene Flagship (Nanocomposites, No. 604391). S.S. acknowledges support from BIHSNAM.

${ }^{1}$ T. Hertel, R. E. Walkup, and P. Avouris, Phys. Rev. B 58, 13870 (1998).

${ }^{2}$ O. Gülseren, T. Yildirim, S. Ciraci, and Ç. Kılıç, Phys. Rev. B 65, 155410 (2002).

${ }^{3}$ T. Tang, A. Jagota, and C.-Y. Hui, J. Appl. Phys. 97, 074304 (2005).

${ }^{4}$ M. C. Strus, L. Zalamea, A. Raman, R. B. Pipes, C. V. Nguyen, and E. A. Stach, Nano Lett. 8, 544 (2008).

${ }^{5}$ M. Ishikawa, R. Harada, N. Sasaki, and K. Miura, Appl. Phys. Lett. 93, 083122 (2008).

${ }^{6}$ M. C. Strus, C. I. Cano, R. B. Pipes, C. V. Nguyen, and A. Raman, Compos. Sci. Technol. 69, 1580 (2009).

${ }^{7}$ M. R. Roenbeck, X. Wei, A. M. Beese, M. Naraghi, A. Furmanchuk, J. T. Paci, G. C. Schatz, and H. D. Espinosa, ACS Nano 8, 124 (2014).

${ }^{8}$ J. Xiao, H. Jiang, D. Y. Khang, J. Wu, Y. Huang, and J. A. Rogers, J. Appl. Phys. 104, 033543 (2008).

${ }^{9}$ N. Sasaki, A. Toyoda, H. Saitoh, N. Itamura, M. Ohyama, and K. Miura, e-J. Surf. Sci. Nanotechnol. 4, 133 (2006).

${ }^{10}$ N. Sasaki, A. Toyoda, N. Itamura, and K. Miura, e-J. Surf. Sci. Nanotechnol. 6, 72 (2008).
${ }^{11}$ J. Buchoux, L. Bellon, S. Marsaudon, and J.-P. Aimé, Eur. Phys. J. B 84, 69 (2011).

${ }^{12}$ D. Y. Khang, J. L. Xiao, C. Kocabas, S. MacLaren, T. Banks, H. Q. Jiang, Y. Y. G. Huang, and J. A. Rogers, Nano Lett. 8, 124 (2008).

${ }^{13}$ M. Ishikawa, R. Harada, N. Sasaki, and K. Miura, Phys. Rev. B 80, 193406 (2009).

${ }^{14}$ G. G. Samsonidze, G. G. Samsonidze, and B. I. Yakobson, Comput. Mater. Sci. 23, 62 (2002).

${ }^{15}$ H. Jiang, X. Q. Feng, Y. Huang, K. C. Hwang, and P. D. Wu, Comput, Methods Appl. Mech. Eng. 193, 3419 (2004).

${ }^{16}$ K. I. Tserpes and P. Papanikos, Compos. Struct. 79, 581 (2007).

${ }^{17}$ G. D. Lee, C. Z. Wang, E. Yoon, N. M. Hwang, and K. M. Ho, Appl. Phys. Lett. 92, 043104 (2008).

${ }^{18}$ E.-L. Florin, M. Rief, H. Lehmann, M. Ludwig, C. Dornmair, V. T. Moy, and H. E. Gaub, Biosens. Bioelectron. 10, 895 (1995).

${ }^{19}$ C. H. Ke, N. Pugno, B. Peng, and H. D. Espinosa, J. Mech. Phys. Solids 53, 1314 (2005)

${ }^{20}$ C. Ke, M. Zheng, G. Zhou, W. Cui, N. Pugno, and R. N. Miles, Small 6, 438 (2010).

${ }^{21}$ C. Ke, M. Zheng, I.-T. Bae, and G. Zhou, J. Appl. Phys. 107, 104305 (2010).

${ }^{22}$ C. H. Ke and H. D. Espinosa, Small 2, 1484 (2006).

${ }^{23}$ T. C. Chu, R. F. Ranson, M. A. Sutton, and M. H. Peters, Exp. Mech. 25, 232 (1985).

${ }^{24}$ Y. Mikata, Acta Mech. 190, 133 (2007).

${ }^{25}$ M. R. Falvo, G. J. Clary, R. M. Taylor, V. Chi, F. P. Brooks, S. Washburn, and R. Superfine, Nature 389, 582 (1997).

${ }^{26}$ Q. Wei, M. Zheng, and C.-H. Ke, Nanosci. Nanotechnol. Lett. 2, 308 (2010). 